\title{
ARTICLE
}

Epidemiology

\section{Type 2 diabetes mellitus, blood cholesterol, triglyceride and colorectal cancer risk in Lynch syndrome}

\author{
S. Ghazaleh Dashti ${ }^{1,2,3}$, Wing Yan $\mathrm{Li}^{1,2}$, Daniel D. Buchanan $\mathbb{D}^{1,2,4,5}$, Mark Clendenning ${ }^{2,5}$, Christophe Rosty ${ }^{2,5,6,7}$, Ingrid M. Winship ${ }^{4,8}$, \\ Finlay A. Macrae ${ }^{4,8,9}$, Graham G. Giles ${ }^{1,3}$, Sheetal Hardikar ${ }^{10,11,12}$, Xinwei Hua ${ }^{12,13}$, Stephen N. Thibodeau ${ }^{14}$, Jane C. Figueiredo ${ }^{15,16}$, \\ Graham Casey $^{17}$, Robert W. Haile ${ }^{15}$, Steven Gallinger ${ }^{18}$, Loïc Le Marchand ${ }^{19}$, Polly A. Newcomb ${ }^{12,13}$, John D. Potter ${ }^{12,13,20}$, \\ Noralane M. Lindor ${ }^{21}$, John L. Hopper ${ }^{1,2}$, Mark A. Jenkins ${ }^{1,2}$ and Aung Ko Win $\mathbb{D}^{1,2,4}$
}

BACKGROUND: Type 2 diabetes mellitus and high total cholesterol and triglycerides are known to be associated with increased colorectal cancer risk for the general population. These associations are unknown for people with a germline DNA mismatch repair gene mutation (Lynch syndrome), who are at high risk of colorectal cancer.

METHODS: This study included 2023 (56.4\% female) carriers with a mismatch repair gene mutation (737 in MLH1, 928 in MSH2, 230 in MSH6, 106 in PMS2, 22 in EPCAM) recruited by the Colon Cancer Family Registry between 1998 and 2012. Weighted Cox regression was used to estimate the hazard ratios (HR) and $95 \%$ confidence intervals (CI) for the associations between self-reported type 2 diabetes, high cholesterol, triglyceride and colorectal cancer risk.

RESULTS: Overall, 802 carriers were diagnosed with colorectal cancer at a median age of 42 years. A higher risk of colorectal cancer was observed in those with self-reported type-2 diabetes (HR 1.92; 95\% Cl, 1.03-3.58) and high cholesterol (HR 1.76; $\mathrm{Cl} 1.23-2.52)$ compared with those without these conditions. There was no evidence of high triglyceride being associated with colorectal cancer risk.

CONCLUSION: For people with Lynch syndrome, self-reported type-2 diabetes mellitus and high cholesterol were associated with increased colorectal cancer risk.

British Journal of Cancer (2019) 121:869-876; https://doi.org/10.1038/s41416-019-0580-9

\section{INTRODUCTION}

Lynch syndrome is caused by a germline mutation in one of the DNA mismatch repair (MMR) genes $M L H 1, M S H 2, M S H 6$ and PMS2 or a deletion in EPCAM. ${ }^{1-5}$ Lynch syndrome is estimated to be present in one in 279 people in the general population ${ }^{6}$ and to cause $\sim 2-5 \%$ of all colorectal cancers. ${ }^{7,8}$ People with Lynch syndrome are at increased risk of various types of cancers, mainly colorectal cancer. The average cumulative risk of colorectal cancer to age 70 years $(95 \%$ confidence interval $(\mathrm{Cl}))$, for male and female mutation carriers, respectively, has been estimated to be $34 \%$ $(25-50 \%)$ and $36 \%(25-51 \%)$ for $M L H 1,{ }^{9}$ and $47 \%(36-60 \%)$ and $37 \%(27-50 \%)$ for $\mathrm{MSH}_{2}{ }^{9} 22 \%(14-32 \%)$ and $10 \%(5-17 \%)$ for
$\mathrm{MSH}^{10}{ }^{10}$ and $7.2 \%(4.5-13 \%)$ and $6.4 \%$ (3.8-12\%) for PMS2. ${ }^{11}$ Further, there is evidence that the risk varies substantially even across carriers of a mutation in the same gene, with the majority of carriers being either at only modestly increased risk or at very high risk, rather than being clustered around the 'average' risk. These findings are consistent with that other genetic or environmental and lifestyle factors may modify cancer risk in people with Lynch syndrome. ${ }^{9,12}$

Previous epidemiological studies have shown that, for the general population, diabetes mellitus, particularly type 2 , is associated with an increased risk of colorectal cancer. ${ }^{13,14}$ Similarly, there is evidence that high blood levels of total

\footnotetext{
${ }^{1}$ Centre for Epidemiology and Biostatistics, Melbourne School of Population and Global Health, The University of Melbourne, Parkville, VIC 3010 , Australia; ${ }^{2}$ Victorian Comprehensive Cancer Centre, University of Melbourne Centre for Cancer Research, Melbourne, VIC 3000, Australia; ${ }^{3}$ Cancer Epidemiology and Intelligence Division, Cancer Council Victoria, Melbourne, VIC 3004, Australia; ${ }^{4}$ Genetic Medicine, Royal Melbourne Hospital, Parkville, VIC 3050, Australia; ${ }^{5}$ Colorectal Oncogenomics Group, Department of Clinical Pathology, The University of Melbourne, Parkville, VIC 3010, Australia; ${ }^{6}$ Envoi Specialist Pathologists, Brisbane, QLD 4059, Australia; ${ }^{7}$ Faculty of Medicine, University of Queensland, Brisbane, QLD 4006, Australia; ${ }^{8}$ Department of Medicine, The University of Melbourne, Parkville, VIC 3010, Australia; ${ }^{9}$ Colorectal Medicine and Genetics, Royal Melbourne Hospital, Parkville, VIC 3050, Australia; ${ }^{10}$ Department of Population Health Sciences, University of Utah, Salt Lake City, UT 84112, USA; ${ }^{11}$ Population Sciences, Huntsman Cancer Institute, Salt Lake City, UT 84112, USA; ${ }^{12}$ Public Health Sciences Division, Fred Hutchinson Cancer Research Center, Seattle, WA 98109, USA; ${ }^{13}$ School of Public Health, University of Washington, Seattle, WA 98195, USA; ${ }^{14}$ Molecular Genetics Laboratory, Department of Laboratory Medicine and Pathology, Mayo Clinic, Rochester, MN 55455, USA; ${ }^{15}$ Samuel Oschin Comprehensive Cancer Institute, Cedars-Sinai Medical Center, Los Angeles, CA 90048, USA; ${ }^{16}$ Department of Preventive Medicine, Keck School of Medicine, University of Southern California, Los Angeles, CA 90032, USA; ${ }^{17}$ Center for Public Health Genomics, University of Virginia, Charlottesville, VA 22908, USA; ${ }^{18}$ Lunenfeld Tanenbaum Research Institute, Mount Sinai Hospital, University of Toronto, Toronto, ON M5G 1×5, Canada; ${ }^{19}$ University of Hawaii Cancer Center, Honolulu, Hawaii 96813, USA; ${ }^{20}$ Centre for Public Health Research, Massey University, Wellington 6140, New Zealand and ${ }^{21}$ Department of Health Science Research, Mayo Clinic Arizona, Scottsdale, AZ 85259, USA
}

Correspondence: Aung Ko Win (awin@unimelb.edu.au)

These authors contributed equally: S. Ghazaleh Dashti, Wing Yan Li

Received: 3 May 2019 Revised: 26 August 2019 Accepted: 30 August 2019

Published online: 25 September 2019 
cholesterol and triglyceride increase the risk of colorectal cancer. ${ }^{15,16}$ Investigating whether such associations exist for people with Lynch syndrome is important for predicting risk for targeted screening and a better understanding of carcinogenesis and could provide opportunities to reduce cancer risk in this highrisk population. In the current study, we report the associations between self-reported diagnosis of diabetes mellitus, high cholesterol and high triglyceride, and the risk of colorectal cancer for people with Lynch syndrome, using a large dataset from the Colon Cancer Family Registry.

\section{MATERIALS AND METHODS}

Study sample

The study sample included carriers of heterozygous germline pathogenic mutations in MMR genes recruited by the Colon Cancer Family Registry (CCFR) between 1998 and 2012. Detailed description of the study design and recruitment by each site of the CCFR have been previously published ${ }^{17,18}$ and can be found at http://www.coloncfr.org/. Briefly, the CCFR recruitment protocols fall broadly into two main categories: population-based and clinicbased. Through population-based cancer registries in the United States (Hawaii, Minnesota, Puget Sound, California, Arizona, Colorado, New Hampshire, and North Carolina), Australia (Victoria) and Canada (Ontario), individuals with recent diagnosis of colorectal cancer were recruited regardless of family histories or stratified by family histories. Through familial cancer clinics in the United States (Mayo Clinic, Rochester, Minnesota, and Cleveland Clinic, Cleveland, Ohio), Canada (Ontario), Australia (Brisbane, Melbourne, Adelaide, Sydney, and Perth), and New Zealand (Auckland), individuals with or without colorectal cancer were recruited because they were part of families with colorectal cancer. Relatives of probands were recruited according to prespecified rules of recruiting centres, with prior permission obtained from the probands. ${ }^{17}$ Informed consent was obtained from all study participants and the study protocol was approved by the research ethics review boards of each recruitment site.

\section{Data collection}

At the time of recruitment, information on demographics and personal characteristics such as education, height, current weight and weight at age 20, lifestyle factors such as smoking, alcohol consumption, physical activity, diet, medical history including having received diagnosis of diabetes mellitus, high cholesterol, and high triglyceride, age at diagnosis of these conditions and any medication used to control them, medication history such as aspirin, calcium supplement intake, cancer screening history, and any previous surgery were obtained from all participating probands and relatives. The questionnaire was standardised across all CCFR sites and was administered via in-person interviews, telephone interviews, or mailed paper questionnaires (questionnaire details can be found at http://coloncfr.org/ questionnaires). Personal and family history of all cancers (except non-melanoma skin cancers) and age diagnosis were also collected. When possible, cancer diagnoses were verified using pathology reports, medical records, and/or cancer registries. Blood samples were collected from all consenting participants and tumour tissues from those diagnosed with colorectal cancer.

\section{MMR gene mutation testing}

Screening for germline mutations in $\mathrm{MLH1}, \mathrm{MSH} 2, \mathrm{MSH}$, PMS2 and EPCAM was performed for all population-based probands who had a colorectal tumour displaying high levels of microsatellite instability (MSI) or a loss of expression of one or more of the MMR proteins by immunohistochemistry $(\mathrm{IHC})$ and, for the youngest-onset colorectal cancer-affected participant from each clinic-based family, regardless of MSI or MMR-protein expression status. ${ }^{17}$ MMR gene mutation testing has been described in detail previously. ${ }^{6}$ Where available, blood samples from the relatives of probands with a pathogenic mutation ${ }^{6}$ were tested for the specific MMR gene mutation identified in the proband (predictive testing).

\section{Definitions of exposures}

The exposures of interest were diabetes mellitus, defined as present if a participant answered "yes" to "has a doctor ever told you that you had diabetes (excluding diabetes which you had only during pregnancy)?"; high cholesterol, defined as present if a participant answered "yes" to "has a doctor ever told you that you had high cholesterol?"; and high triglycerides defined as present if a participant answered "yes" to "has a doctor ever told you that you have high levels of triglycerides in your blood?". Ages at diagnoses of these conditions were self-reported. The three exposure variables were treated as time-varying exposures, e.g. carriers were categorised as not having diabetes prior to age of diagnosis of diabetes mellitus and as having diabetes mellitus at age of diagnosis of diabetes mellitus and thereafter. Exposure variables were coded as missing if reported age at diagnosis of the condition was missing (two participants with diabetes mellitus, 28 with high cholesterol and 15 with high triglyceride) and as zero if age at diagnosis was after the age at colorectal cancer diagnosis or age at censoring (see below). Information on type of diabetes mellitus was not collected. However, we attempted to identify carriers with type 2 diabetes mellitus by defining them as those diagnosed with diabetes mellitus at age 35 or older, or diagnosed with diabetes mellitus under age 35 and on oral medication. ${ }^{19}$

\section{Statistical analysis}

We included probands and relatives with confirmed pathogenic MMR gene mutations-from both clinic-based and populationbased CCFR sites-who had completed the interview at recruitment. For this study, all analyses were restricted to data for exposures, outcome, and covariates collected at the time of recruitment, i.e. at age at interview.

We conducted weighted Cox proportional hazards regression analysis, with age as the time scale, to estimate the association between diabetes mellitus, high cholesterol, and high triglyceride with the risk of colorectal cancer. Time at risk started at age 20 and ended at age at first diagnosis of colorectal cancer, age at polypectomy, diagnosis of non-skin, non-colorectal cancers (excluding non-melanoma skin cancers), or age at interview, whichever occurred first. Diabetes mellitus, high cholesterol, and high triglycerides were fitted as time-varying exposures in the models. Data on self-reported age at diagnosis of the three primary exposures variables was used to define time-varying exposure variables; therefore, exposure time started at age at diagnosis of each condition.

Because some participants were recruited from families with colorectal cancer histories and case probands were tested preferentially for MMR gene mutations, participants included in the analysis were not randomly ascertained in relation to disease status. To minimise bias caused by this non-random ascertainment, we used a "weighted cohort approach" described by Antoniou et al., ${ }^{20}$ which has been used in similar studies of modifiers for Lynch syndrome. ${ }^{21-26}$ Briefly, we first calculated the incidence rates of colorectal cancer for the MMR mutation carriers for age groups <30, 30-34, 35-39, 40-44, 45-49, 50-54, 55-59, 60-64 and >64 based on colorectal cancer incidence in the general population, averaged for men and women, ${ }^{27}$ and the hazard ratio of colorectal cancer for each MMR gene mutated. ${ }^{9,10,28}$ These incidence rates was then used together with the number and total person-years of observation for affected and unaffected carriers in each age group to calculate the age-specific sampling fractions. Finally, probability weights were calculated based on the sampling fractions and assigned to the affected and unaffected carriers, so that the age-specific proportion of affected carriers in the "synthetic" cohort equalled the proportion 
Table 1. Characteristics of people with Lynch syndrome included in the study

\begin{tabular}{|c|c|c|c|}
\hline & $\begin{array}{l}\text { No colorectal cancer } \\
(N=1221)\end{array}$ & $\begin{array}{l}\text { Colorectal cancer } \\
(N=802)\end{array}$ & $\begin{array}{l}\text { Overall } \\
(N=2023)\end{array}$ \\
\hline \multicolumn{4}{|l|}{ Sex, $n(\%)$} \\
\hline Female & $776(63.6)$ & $365(45.5)$ & $1141(56.4)$ \\
\hline \multicolumn{4}{|l|}{ Study Centres, $n$ (\%) } \\
\hline USA & $341(27.9)$ & $294(36.7)$ & $635(31.4)$ \\
\hline \multicolumn{4}{|l|}{ Ascertainment method, $n$ (\%) } \\
\hline Clinic & $968(79.3)$ & $524(65.3)$ & $1492(73.8)$ \\
\hline Population & $253(20.7)$ & $278(34.7)$ & $531(26.2)$ \\
\hline \multicolumn{4}{|l|}{ Proband, $n(\%)$} \\
\hline [range] & {$[20-85]$} & {$[20-76]$} & {$[20-85]$} \\
\hline \multicolumn{4}{|l|}{ Education level, $n$ (\%) } \\
\hline Some high school or less & $242(19.8)$ & $189(23.6)$ & $431(21.3)$ \\
\hline Completed high school/some tertiary study & $404(33.1)$ & $268(33.4)$ & $672(33.2)$ \\
\hline Vocational/technical school & $223(18.3)$ & $126(15.7)$ & $349(17.3)$ \\
\hline University degree & $338(27.7)$ & $205(25.6)$ & $543(26.8)$ \\
\hline Missing & $14(1.1)$ & $14(1.7)$ & $28(1.4)$ \\
\hline \multicolumn{4}{|l|}{ Mismatch repair gene mutated, $n$ (\%) } \\
\hline MLH1 & 389 (31.9) & $348(43.4)$ & 737 (36.4) \\
\hline $\mathrm{MSH} 2$ & $600(49.1)$ & $328(40.9)$ & $928(45.9)$ \\
\hline$\geq 30 \mathrm{~kg} / \mathrm{m}^{2}$ & $49(4.0)$ & $43(5.4)$ & $92(4.5)$ \\
\hline Missing & $49(4.0)$ & $36(4.5)$ & $85(4.2)$ \\
\hline \multicolumn{4}{|l|}{ Regular physical activity, ${ }^{\mathrm{a}} n(\%)$} \\
\hline No & $53(4.3)$ & $49(6.1)$ & $102(5.0)$ \\
\hline Yes & $1,128(92.4)$ & $713(88.9)$ & $1841(91.0)$ \\
\hline Missing & $40(3.3)$ & $40(5.0)$ & $80(4.0)$ \\
\hline \multicolumn{4}{|l|}{ Cigarette smoking, ${ }^{\mathrm{b}} \mathrm{n}(\%)$} \\
\hline Never smoker & $667(54.6)$ & $359(44.8)$ & $1026(50.7)$ \\
\hline Former smoker & $274(22.4)$ & $170(21.2)$ & $444(21.9)$ \\
\hline Current smoker & $275(22.5)$ & $271(33.8)$ & $546(27.0)$ \\
\hline Missing & $5(0.4)$ & $2(0.2)$ & $7(0.3)$ \\
\hline \multicolumn{4}{|l|}{ Average daily ethanol intake (grams/day), ${ }^{\mathrm{C}} n(\%)$} \\
\hline 0 & $321(26.3)$ & $200(24.9)$ & $521(25.8)$ \\
\hline$>0-\leq 14$ & $580(47.5)$ & $314(39.2)$ & $894(44.2)$ \\
\hline$>14-\leq 28$ & $113(9.3)$ & $85(10.6)$ & $198(9.8)$ \\
\hline$>28$ & $89(7.3)$ & $111(13.8)$ & $200(9.9)$ \\
\hline Missing & $118(9.7)$ & $92(11.5)$ & $210(10.4)$ \\
\hline \multicolumn{4}{|c|}{ Fruit and vegetable intake (servings/day) 2 years before diagnosed/censored age, ${ }^{d, f} n(\%)$} \\
\hline$<2$ & $200(16.4)$ & $123(15.3)$ & $323(16.0)$ \\
\hline $2.01-3$ & $141(11.5)$ & $54(6.7)$ & $195(9.6)$ \\
\hline
\end{tabular}




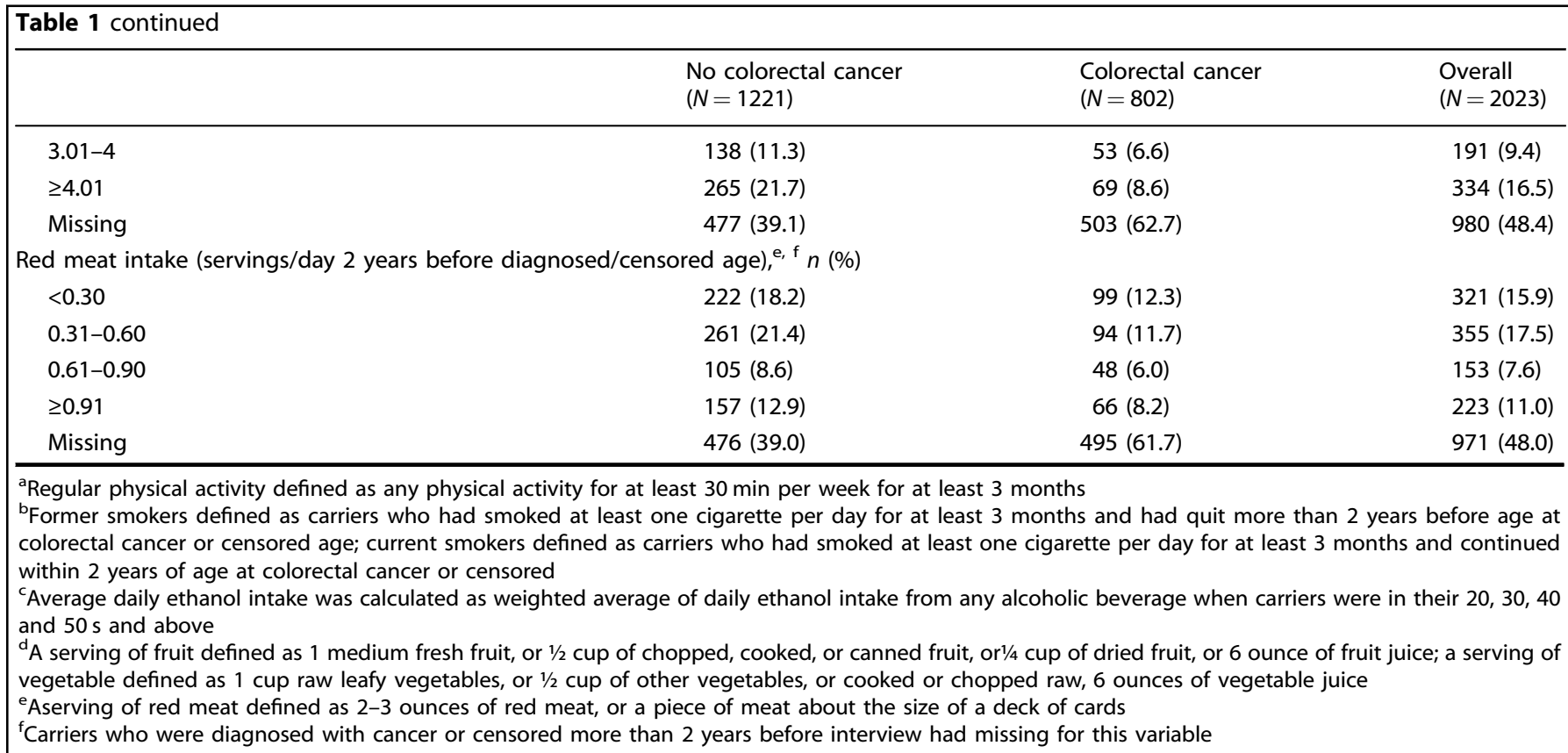

of affected carriers in the general population. ${ }^{29}$ The Huber-White robust variance estimation by clustering on family membership was applied to account for possible correlation in risk between carriers from the same family. ${ }^{30}$

Sex, ascertainment method, country of recruitment, education, body mass index (BMI) at age 20, average daily ethanol intake from alcoholic beverages, smoking status, and physical activity were identified as potential confounders. When applicable, potential confounders were also fitted as time-varying covariates. Diabetes mellitus was also included as a confounder in multivariable models treating high cholesterol or high triglyceride as the exposure. We did not condition on high cholesterol or high triglyceride in the models for diabetes mellitus, because these might have been on the causal pathway from diabetes mellitus to colorectal cancer incidence. ${ }^{31}$ Fruit, vegetable and meat intake were not included in the primary analysis, because carriers who were censored or received colorectal cancer diagnosis more than 2 years before interview were missing information for those variables. To test for interactions, the difference in the loglikelihood ratio was assessed after adding a cross-product term between each exposure variable and the potential effect modifiers identified a priori (carrier's sex and mutated MMR gene).

The number of missing values for all variables are reported in Tables 1 and 2. All analyses were complete case analyses. We did not test for the proportional hazards assumption, because it cannot be interpreted with time-varying exposure.

We also undertook additional analyses restricted to carriers who had a colorectal cancer diagnosis or were censored within 5 years before interview; analyses additionally adjusted for consumptions of fruit, vegetable and red meat intake 2 years before colorectal cancer diagnosis or censoring; and analyses without applying the probability weights.

All $p$-values reported are two-sided. Stata version 14.2 was used for statistical analysis. ${ }^{32}$

\section{RESULTS}

A total of 2164 MMR gene mutation carriers were identified across the CCFR. Of these, 65 who had not completed the interview at baseline recruitment, six with missing age at cancer diagnosis, 13 who did not have data on diabetes mellitus, high cholesterol and high triglyceride and 57 who were censored before age 20 were excluded. The final sample comprised 2023 carriers; of whom 694 (34.3\%) were probands; 1141 (56.4\%) were females, 1492 (73.8\%) were recruited from familial cancer clinics; 737 carried a mutation in MLH1, 928 in MSH2, 230 in MSH6, 106 in PMS2, and 22 in EPCAM. Participants were from 773 families, of which 431 had one affected relative, 148 had more than one affected relative, and 194 had no affected relative. The characteristics of people with Lynch syndrome included in the study are summarised in Table 1. For 802 of the included people, time at risk ended at colorectal cancer diagnosis, for 344 at first polypectomy, for 267 at non-skin noncolorectal cancers diagnosis, and for 610 at interview. The median ages (interquartile range) at colorectal cancer diagnosis and censoring were 42 (35-49) and 41 (32-50) years, respectively. Incidence rate of colorectal cancer from birth was $943(95 \%$ confidence interval (Cl) 880-1011) per 100,000 person-years.

Overall, 66 (3.3\%) carriers reported having been diagnosed with diabetes mellitus before colorectal cancer diagnoses or censoring (median (interquartile) age at diagnosis 50 (42-60) years). Of these, $60(91 \%)$ were categorised as having type 2 mellitus (the remaining $9 \%$ were not included in analyses that compared colorectal cancer risk in carriers with versus without type 2 diabetes). A total of 250 (12.4\%) carriers reported having been diagnosed with high blood cholesterol at a median (interquartile) age of $50(41-58)$ years, and $81(4.0 \%)$ reported having been diagnosed with high triglyceride at a median (interquartile) age of 48 (40-55) years (Table 2).

After adjusting for potential confounders, carriers with type 2 diabetes mellitus had a higher risk of colorectal cancer compared with those without diabetes mellitus (HR 1.92; $95 \%$ $\mathrm{Cl}, 1.03-3.58)$. There was also evidence of a positive association between high blood cholesterol and the risk of colorectal cancer (HR 1.76; 95\% Cl 1.23-2.52). We did not observe evidence for an association between blood triglyceride and colorectal cancer risk for people with Lynch syndrome in the adjusted model (HR 1.42; 95\% Cl 0.77-2.64) (Table 3). Results were similar when analyses were limited to carriers who reported not taking any medication to control their high cholesterol or high triglyceride (Table 3). The directions and strength of associations were similar when analyses were restricted to carriers censored within 5 years before baseline interview (Supplementary Table 1). There 
Type 2 diabetes mellitus, blood cholesterol, triglyceride and colorectal... SG Dashti et al.

Table 2. Diabetes mellitus, high cholesterol, and high triglyceride levels in people with Lynch syndrome included in the study

\begin{tabular}{|c|c|c|c|}
\hline & No colorectal cancer $(N=1221)$ & Colorectal cancer $(N=802)$ & Overall $(N=2023)$ \\
\hline No & $1182(96.8)$ & $761(94.9)$ & $1943(96.0)$ \\
\hline Yes, type $2^{\mathrm{a}}$ & $30(2.5)$ & $30(3.7)$ & $60(3.0)$ \\
\hline Missing & $6(0.5)$ & $8(1.0)$ & $14(0.7)$ \\
\hline \multicolumn{4}{|c|}{ Medication to control diabetes, $n$ (\% of diabetics) } \\
\hline Insulin injection only & $6(18.2)$ & $6(18.2)$ & $12(18.2)$ \\
\hline Pills and insulin injections & $6(18.2)$ & $11(33.3)$ & $17(25.8)$ \\
\hline \multicolumn{4}{|c|}{ Age at diagnosis of diabetes mellitus (year) } \\
\hline Median (interquartile range) & $51(44-61)$ & $50(40-58)$ & $50(42-60)$ \\
\hline \multicolumn{4}{|c|}{ Years from diabetes diagnosis to colorectal cancer diagnosis or censoring } \\
\hline Missing & $21(1.7)$ & $15(1.9)$ & $36(1.8)$ \\
\hline \multicolumn{4}{|c|}{ Medication to control high cholesterol, $n$ (\% of with high cholesterol) } \\
\hline No & $80(50.0)$ & $47(52.2)$ & $127(50.8)$ \\
\hline Yes & 79 (49.4) & $43(47.8)$ & $122(48.8)$ \\
\hline Missing & $1(0.6)$ & 0 & $1(0.4)$ \\
\hline \multicolumn{4}{|c|}{ Age at diagnosis of high cholesterol (year) } \\
\hline Median (interquartile range) & $50(41-58.5)$ & $50(40-58)$ & $50(41-58)$ \\
\hline \multicolumn{4}{|c|}{ Years from high cholesterol diagnosis to colorectal cancer diagnosis or censoring } \\
\hline Median (interquartile range) & $4(1-9)$ & $4(1-9)$ & $4(1-9)$ \\
\hline \multicolumn{4}{|l|}{ High triglyceride, $n(\%)$} \\
\hline No & $1111(91.0)$ & $729(90.9)$ & $1840(91.0)$ \\
\hline \multicolumn{4}{|c|}{ Years from high triglyceride diagnosis to colorectal cancer diagnosis or censoring } \\
\hline Median (interquartile range) & $49(1-8)$ & $4(1-9)$ & $4(1-9)$ \\
\hline
\end{tabular}

was no statistical evidence for an interaction between these exposure variables and sex or mutated MMR genes (Supplementary Table 2).

Analyses that were additionally adjusted for fruit, vegetable and red meat intake 2 years before colorectal cancer diagnosis or censoring yielded results comparable with those from the primary analysis (detailed results not shown). Results from unweighted analyses were similar to those from weighted analyses, although the standard errors were smaller, and the $95 \%$ Cls were narrower in the former (detailed results not shown).

\section{DISCUSSION}

In this study of people with Lynch syndrome, we observed a higher risk of colorectal cancer associated with self-reported diagnosis of type 2 diabetes mellitus and high blood cholesterol compared with those without these conditions. There was no evidence that these associations differed by sex or the mutated MMR gene.
A meta-analysis of 30 prospective studies reported that diabetes mellitus (most studies did not distinguish the type) increased the risk of colorectal cancer in the general population (summary RR 1.27; $95 \% \mathrm{Cl} 1.21-1.34) .{ }^{14}$ Our estimated $95 \%$ confidence interval overlapped with what was estimated by the meta-analysis, suggesting that the increased risk of colorectal cancer for people with Lynch syndrome is likely to be comparable with that for the general population. In this study, we had a limited number of people with type 2 diabetes mellitus (66 overall (3.3\%) and 33 with colorectal cancer), which might explain our wide confidence interval. Low prevalence of diabetes in our relatively young cohort (median age at colorectal cancer diagnosis or censoring was 42 years (interquartile range 33-50)) was comparable with the under $5 \%$ prevalence reported in people younger than 45 -years-old in the USA, ${ }^{33}$ Canada $^{34}$ and Australia. ${ }^{35}$

For the general population, several underlying mechanisms have been proposed to explain the role of diabetes mellitus on cancer risk. ${ }^{36-38} \mathrm{~A}$ characteristic of type 2 diabetes mellitus is insulin resistance and hyperinsulinemia. Insulin may increase the 
Table 3. Hazard ratios for association between diabetes mellitus, high cholesterol, high triglyceride and colorectal cancer risk in people with Lynch syndrome included in the study

\begin{tabular}{|c|c|c|c|c|c|c|}
\hline & No. Cases & Person-years & \multicolumn{2}{|c|}{ Univariable analysis } & \multicolumn{2}{|c|}{ Multivariable analysis } \\
\hline \multicolumn{7}{|l|}{ Diabetes mellitus } \\
\hline No & 761 & 43653 & 1 (Ref) & & 1 (Ref) & \\
\hline Yes, type 1 and type 2 & 33 & 560 & $2.27(1.41-3.64)$ & 0.001 & $1.71(0.98-2.97)$ & 0.06 \\
\hline Yes, classified as type $2^{\mathrm{a}}$ & 30 & 447 & $2.42(1.46-4.03)$ & 0.001 & $1.92(1.03-3.58)$ & 0.04 \\
\hline Yes & 90 & 1849 & $1.51(1.09-2.10)$ & 0.01 & $1.76(1.23-2.52)$ & 0.03 \\
\hline Yes, and not taking any medication to control high cholesterol & 47 & 670 & $2.16(1.45-3.21)$ & $<0.001$ & $2.59(1.65-4.05)$ & $<0.001$ \\
\hline \multicolumn{7}{|l|}{ High triglyceride $^{\mathrm{b}}$} \\
\hline No & 729 & 41396 & 1 (Ref) & & 1 (Ref) & \\
\hline Yes & 32 & 589 & $1.72(0.99-2.98)$ & 0.05 & $1.42(0.77-2.64)$ & 0.26 \\
\hline
\end{tabular}

risk of cancer through direct and indirect (via increased free insulin-like growth factor-1) mitogenic and anti-apoptotic properties. ${ }^{36}$ Hyperglycaemia might also increase cancer risk as glucose provides fuel to cancer cells and increases oxidative stress and inflammatory cytokines. ${ }^{39}$ The colorectal carcinogenesis is complex, heterogenous and associated with multiple factors including genetic, epigenetic and environmental factors. Most of Lynch syndrome-related colorectal cancers developed through the MSI pathway which may affect genes that control cell growth (transforming growth factor [TGF] beta and insulinlike growth factor [sIGF] receptors), regulate apoptotic cell death (Caspase 5, Bax), and some of the DNA MMR genes themselves (MSH3, MSH6). Accumulation of mutations in these cancerrelated genes is assumed to drive the carcinogenesis in Lynch syndrome. Evidence suggesting that the effect of type 2 diabetes mellitus would be different on tumours with and without MSI-high is currently lacking. However, a study of 1116 stage-II colon cancer cases observed diabetes mellitus in a comparable proportion of cases with and without MSI-high tumours. ${ }^{40}$

A mendelian randomisation study has shown an increased risk of colorectal cancer with genetically determined higher levels of total cholesterol (odds ratio (OR) per unit increase in genetic risk score 1.46; $95 \% \mathrm{Cl}, 1.20-1.79)$ for the general population. ${ }^{16}$ Previous epidemiological studies have also reported a higher risk of colorectal cancer in association with high versus low levels of total cholesterol (summary RR from a pooled analysis of 10 prospective studies $1.11 ; 95 \% \mathrm{Cl} 1.01-1.21) .{ }^{15}$ Our estimated $95 \%$ confidence interval for people with Lynch syndrome from this study overlapped with that with that of the pooled estimate. The mechanisms linking high cholesterol levels to colorectal cancer risk remain speculative; it is suggested that cholesterol may exert carcinogenic effects by increasing oxidative stress and inflammation and compromising immunosurveillance. ${ }^{16}$

High blood triglyceride has been reported to be associated with an increased risk of colorectal cancer for the general population (summary RR from a pooled analysis of 9 prospective studies 1.18;

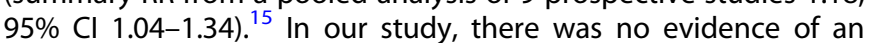
association between high triglyceride and colorectal cancer risk
(HR 1.07; 95\% Cl 0.55-2.09). However, the absence of evidence in our study might have been due to lack of sufficient statistical power to identify any association.

To the best of our knowledge, this is the first study to date assessing the associations between diabetes mellitus, high blood cholesterol and triglyceride and colorectal cancer risk for people with Lynch syndrome. Data for this study were collected using standardised questionnaires and protocols and comprehensive high-quality genetic testing was undertaken to identify MMR mutation carriers in the CCFR. ${ }^{17}$ Self-reported age at diagnosis of the three primary exposures allowed us to analyse these variables as time-varying and thus minimise immortal time bias. ${ }^{41}$ The application of a weighted cohort approach reduced the ascertainment bias of mutation carriers on the basis of their disease status or being part of colorectal cancer families. ${ }^{20}$

A limitation of our study was possible error in measurements of exposures (and other confounding variables), because these were based on self-reported questionnaires. A study of 2037 residents in Minnesota has provided evidence on a substantial agreement (over 95\%) between self-reported diabetes mellitus status and medical records. ${ }^{42}$ This agreement may be lower for high cholesterol and triglycerides. ${ }^{43}$ Because the current study included data from the baseline interview at which study participants were aware of their cancer status, this measurement error might have been differential with respect to outcome (recall bias), biasing the results towards or away from the null. Since information on the type of diabetes mellitus was not collected in the CCFR, we classified the type using the self-reported age at diagnosis and type of medication used for diabetes control, based on suggested criteria in a systematic review on clinical characteristic for differentiating type 1 and type 2 diabetes. ${ }^{19}$ Using this approach, $91 \%$ of our study participants with diabetes mellitus were classified as having type 2 diabetes, which is consistent with the prevalence in the population. ${ }^{44}$

We explored the potential impact of survival bias on our results by performing additional analyses restricted to carriers who had a cancer diagnosed or were censored within 5 years before baseline interview. Findings from these analyses were not different from the primary analyses. Another limitation of this study is the 
Type 2 diabetes mellitus, blood cholesterol, triglyceride and colorectal... SG Dashti et al.

possibility of residual confounding by factors that we were not able to adjust for in the analyses. We were not able to include fruit, vegetable, and red meat intake in our primary analysis, because carriers who were diagnosed with colorectal cancer or censored more than 2 years before interview had missing values for these variables (the questionnaire asked about these factors 2 years before interview). When these variables were adjusted for in multivariable models as sensitivity analyses, the estimates did not substantially change in direction and strength, compared with those from primary analysis. Although we did not have sufficient statistical power to investigate the association between the exposure variables and colorectal cancer risk by the mutated MMR gene, our test for interaction provided no evidence of effect modification by the mutated gene.

In summary, our study provides evidence that self-reported type 2 diabetes and high cholesterol are associated with an increased risk of colorectal cancer in Lynch syndrome. These associations do not appear to be different across the mutated MMR genes or between male and female carriers. Our finding is consistent with that these are additional risk factors that could be fruitfully monitored and controlled in people with Lynch syndrome, who are at high risk of colorectal cancer.

\section{ACKNOWLEDGEMENTS}

We thank all study participants of the CCFR and staff for their contributions to this project. The content of this paper does not necessarily reflect the views or policies of the National Cancer Institute or any of the collaborating sites in the CCFR, nor does mention of trade names, commercial products, or organisations imply endorsement by the US Government or the CCFR.

\section{AUTHOR CONTRIBUTIONS}

S.G.D., W.Y.L., A.K.W.-study design and concept, acquisition of data, analysis and interpretation of the data, drafting of the paper, approval of the final version of the paper; M.C., C.R., I.M.W., F.A.M., G.G.G., S.H., X.H., S.N.T., J.C.F., G.C., R.W.H., S.G., L.L.M., P.A.N., J.D.P., N.M.L., J.L.H., M.A.J.-acquisition of data, interpretation of the data, critical revision of the paper, approval of the final version of the paper.

\section{ADDITIONAL INFORMATION}

Supplementary information is available for this paper at https://doi.org/10.1038/ s41416-019-0580-9.

Competing interests: The authors declare no competing interests.

Ethics approval and consent to participate: Informed consent was obtained from all study participants and the study protocol was approved by the Human Research Ethics Committees from each recruitment site of the Colon Cancer Family Registry. The study was approved by the Colon Cancer Family Registry Steering Committee (ID C-AU-0208-01). The study was performed in accordance with the Declaration of Helsinki.

Funding: This work was supported by supported by the National Cancer Institute $(\mathrm{NCl})$ of the National Institutes of Health $(\mathrm{NIH})$ under Award Number U01 CA167551 and through $\mathrm{NCl} / \mathrm{NIH}$ cooperative agreements with the following Colon Cancer Family Registry (CCFR) sites: Australasian Colorectal Cancer Family Registry (U01 CA074778 and U01/U24 CA097735), Mayo Colon Cancer Family Registry (U01/U24 CA074800), Ontario Familial Colorectal Cancer Registry (U01/U24 CA074783), Seattle Familial Colorectal Cancer Registry (U01/U24 CA074794), Hawaii Family Registry of Colon Cancer (U01/U24 CA074806 and R01 CA104132 to L LeMarchand), and CedarsSinai Medical Center Consortium (U01/U24 CA074799). Additional support for case ascertainment was provided from the Surveillance, Epidemiology and End Results (SEER) Program of the National Cancer Institute to Fred Hutchinson Cancer Research Center (Control Nos. N01-CN-67009 and N01-PC-35142, and Contract No. HHSN2612013000121), the Hawaii Department of Health (Control Nos. N01-PC67001 and N01-PC-35137, and Contract No. HHSN26120100037C), and the California Department of Public Health (contracts HHSN261201000035C awarded to the University of Southern California and HHSN261201000140C awarded to the Cancer Prevention Institute of California), the following U.S. state cancer registries: $A Z, C O$, $\mathrm{MN}, \mathrm{NC}, \mathrm{NH}$, and by the Victorian Cancer Registry, Australia and the Ontario Cancer
Registry, Canada. This study was also supported by the Australian National Health and Medical Research Council (NHMRC) Fellowships awarded to AKW, DDB (Career Development Fellowships), MAJ (Senior Research Fellowship) and JLH (Senior Principal Research Fellowship). DDB is also funded by a University of Melbourne Research at Melbourne Accelerator Program (R@MAP).

Consent for publish: Not applicable.

Data availability: The data supporting the results reported in this article are available from the Colon Cancer Family Registry (https://www.coloncfr.org/). The data are not publicly available and are subject to data use agreement.

Note: This work is published under the standard license to publish agreement. After 12 months the work will become freely available and the license terms will switch to a Creative Commons Attribution 4.0 International (CC BY 4.0).

Publisher's note Springer Nature remains neutral with regard to jurisdictional claims in published maps and institutional affiliations.

\section{REFERENCES}

1. Bronner, C. E., Baker, S. M., Morrison, P. T., Warren, G., Smith, L. G., Lescoe, M. K et al. Mutation in the DNA mismatch repair gene homologue hMLH 1 is associated with hereditary non-polyposis colon cancer. Nature. 368, 258-261 (1994).

2. Nicolaides, N. C., Papadopoulos, N., Liu, B., Weit, Y.-F., Carter, K. C., Ruben, S. M. et al. Mutations of two PMS homologues in hereditary nonpolyposis colon cancer. Nature. 371, 75-80 (1994).

3. Fishel, R., Lescoe, M., Rao, M., Copeland, N., Jenkins, N., Garber, J. et al. The human mutator gene homolog MSH2 and its associations with hereditary nonpolyposis colon cancer. Cell. 75, 1027-1038 (1993).

4. Akiyama, Y., Sato, H., Yamada, T., Nagasaki, H., Tsuchiya, A., Abe, R. et al. Germ-line mutation of the hMSH6/GTBP gene in an atypical hereditary nonpolyposis colorectal cancer kindred. Cancer Res. 57, 3920-3923 (1997).

5. Ligtenberg, M. J., Kuiper, R. P., Chan, T. L., Goossens, M., Hebeda, K. M., Voorendt, $M$. et al. Heritable somatic methylation and inactivation of MSH2 in families with Lynch syndrome due to deletion of the $3^{\prime}$ exons of TACSTD1. Nature Genet. 41, 112-117 (2009).

6. Win, A. K., Jenkins, M. A., Dowty, J. G., Antoniou, A. C., Lee, A., Giles, G. G. et al. Prevalence and penetrance of major genes and polygenes for colorectal cancer. Cancer Epidemiol. Biomarkers Prev. 26, 404-412 (2017).

7. Aaltonen, L. A., Sankila, R., Mecklin, J. P., Jarvinen, H., Pukkala, E., Peltomaki, P. et al. A novel approach to estimate the proportion of hereditary nonpolyposis colorectal cancer of total colorectal cancer burden. Cancer Detect. Prev. 18, 57-63 (1994).

8. Hopper, J. L. Application of genetics to the prevention of colorectal cancer. Recent Results Cancer Res. 166, 17-33 (2005)

9. Dowty, J. G., Win, A. K., Buchanan, D. D., Lindor, N. M., Macrae, F. A., Clendenning, M. et al. Cancer risks for MLH1 and MSH2 mutation carriers. Hum. Mutat. 34, 490-497 (2013).

10. Baglietto, L., Lindor, N. M., Dowty, J. G., White, D. M., Wagner, A., Gomez Garcia, E. B. et al. Risks of Lynch syndrome cancers for MSH6 mutation carriers. J. Natl Cancer Inst. 102, 193-201 (2010).

11. Ten Broeke, S. W., van der Klift, H. M., Tops, C. M. J., Aretz, S., Bernstein, I., Buchanan, D. D. et al. Cancer risks for PMS2-associated Lynch syndrome. J. Clin. Oncol. 36, 2961-2968 (2018).

12. Win, A. K. \& Scott, R. J. Genetic and Environmental Modifiers of Cancer Risk in Lynch Syndrome. in Hereditary Colorectal Cancer. (eds Valle, L., Gruber, S. B. \& Capellá, G.) 67-89 (Springer International Publishing, Cham, 2018).

13. Tsilidis, K. K., Kasimis, J. C., Lopez, D. S., Ntzani, E. E. \& loannidis, J. P. Type 2 diabetes and cancer: umbrella review of meta-analyses of observational studies. BMJ. 350, g7607 (2015).

14. Jiang, Y., Ben, Q., Shen, H., Lu, W., Zhang, Y. \& Zhu, J. Diabetes mellitus and incidence and mortality of colorectal cancer: a systematic review and metaanalysis of cohort studies. Eur. J. Epidemiol. 26, 863-876 (2011).

15. Yao, X. \& Tian, Z. Dyslipidemia and colorectal cancer risk: a meta-analysis of prospective studies. Cancer Causes Control 26, 257-268 (2015).

16. Rodriguez-Broadbent, H., Law, P. J., Sud, A., Palin, K., Tuupanen, S., Gylfe, A. et al. Mendelian randomisation implicates hyperlipidaemia as a risk factor for colorectal cancer. Int. J. Cancer. 140, 2701-2708 (2017).

17. Newcomb, P. A., Baron, J., Cotterchio, M., Gallinger, S., Grove, J., Haile, R. et al. Colon cancer family registry: an international resource for studies of the genetic 
Type 2 diabetes mellitus, blood cholesterol, triglyceride and colorectal... SG Dashti et al.

epidemiology of colon cancer. Cancer Epidemiol. Biomarkers Prev. 16, 2331-2343 (2007).

18. Jenkins, M. A., Win, A. K., Templeton, A. S., Angelakos, M. S., Buchanan, D. D., Cotterchio, $M$. et al. Cohort profile: the colon cancer family registry cohort (CCFRC). Int. J. Epidemiol. 47, 387-8i (2018).

19. Shields, B. M., Peters, J. L., Cooper, C., Lowe, J., Knight, B. A., Powell, R. J. et al. Can clinical features be used to differentiate type 1 from type 2 diabetes? A systematic review of the literature. BMJ Open. 5, e009088 (2015).

20. Antoniou, A. C., Goldgar, D. E., Andrieu, N., Chang-Claude, J., Brohet, R., Rookus, M. A. et al. A weighted cohort approach for analysing factors modifying disease risks in carriers of high-risk susceptibility genes. Genet. Epidemiol. 29, 1-11 (2005).

21. Ait Ouakrim, D., Dashti, S. G., Chau, R., Buchanan, D. D., Clendenning, M., Rosty, C. et al. Aspirin, ibuprofen, and the risk of colorectal cancer in Lynch syndrome. J. Natl Cancer Inst. 107, djv170 (2015).

22. Dashti, S. G., Chau, R., Ouakrim, D. A., Buchanan, D. D., Clendenning, M., Young, J. $P$. et al. Female hormonal factors and the risk of endometrial cancer in Lynch syndrome. JAMA. 314, 61-71 (2015).

23. Win, A. K., Dowty, J. G., English, D. R., Campbell, P. T., Young, J. P., Winship, I. et al. Body mass index in early adulthood and colorectal cancer risk for carriers and noncarriers of germline mutations in DNA mismatch repair genes. Br. J. Cancer. 105, 162-169 (2011).

24. Pande, M., Lynch, P. M., Hopper, J. L., Jenkins, M. A., Gallinger, S., Haile, R. W. et al. Smoking and colorectal cancer in Lynch syndrome: results from the Colon Cancer Family Registry and the University of Texas M.D. Anderson Cancer Center. Clin. Cancer Res. 16, 1331-1339 (2010).

25. Dashti, S. G., Win, A. K., Hardikar, S. S., Glombicki, S. E., Mallenahalli, S., Thirumurthi, S. et al. Physical activity and the risk of colorectal cancer in Lynch syndrome. Int. J. Cancer. 143, 2250-2260 (2018).

26. Dashti, S. G., Buchanan, D. D., Jayasekara, H., Ait Ouakrim, D., Clendenning, M., Rosty, C. et al. Alcohol consumption and the risk of colorectal cancer for mismatch repair gene mutation carriers. Cancer Epidemiol. Biomarkers Prev. 26, 366-375 (2017).

27. Curado, M. P., Edwards, B., Shin, H. R., Storm, H., Ferlay, J., Heanue, M., et al., editors. Cancer Incidence in Five Continents, Vol. IX. (International Agency for Research on Cancer, Lyon, 2007).

28. Senter, L., Clendenning, M., Sotamaa, K., Hampel, H., Green, J., Potter, J. D. et al. The clinical phenotype of Lynch syndrome due to germ-line PMS2 mutations. Gastroenterology. 135, 419-428 (2008).

29. Antoniou, A. C., Goldgar, D. E., Andrieu, N., Chang-Claude, J., Brohet, R., Rookus, M. A. et al. A weighted cohort approach for analysing factors modifying disease risks in carriers of high-risk susceptibility genes. Genet. Epidemiol. 29, 1-11 (2005).

30. Williams, R. L. A note on robust variance estimation for cluster-correlated data. Biometrics. 56, 645-646 (2000).

31. Wu, L. \& Parhofer, K. G. Diabetic dyslipidemia. Metabolism. 63, 1469-1479 (2014).

32. StataCorp. Stata Statistical Software: Release 14. College Station, TX: StataCorp LP. 2015.

33. Center for Disease Control and Prevention. National Diabetes Statistics Report. (Centers for Disease Control and Prevention, U.S. Dept of Health and Human Services, Atlanta, 2017).

34. Public Health Agency of Canada. Report from the National Diabetes Surveillance System Diabetes in Canada (2009).

35. Australian Bureau of Statistics. Diabetes in Australia: A Snapshot. 2007-2008 (2011).

36. Giovannucci, E., Harlan, D. M., Archer, M. C., Bergenstal, R. M., Gapstur, S. M., Habel, L. A. et al. Diabetes and cancer: a consensus report. CA: Cancer J. Clin. 60 , 207-221 (2010).

37. Pollak, $M$. The insulin and insulin-like growth factor receptor family in neoplasia: an update. Nat Rev. Cancer 12, 159-169 (2012).

38. Giovannucci, E. Insulin, insulin-like growth factors and colon cancer: a review of the evidence. J. Nutr. 131(11 Suppl), 3109S-3120SS (2001).

39. Chang, S. C. \& Yang, W. V. Hyperglycemia, tumorigenesis, and chronic inflammation. Crit. Rev. Oncol. Hematol. 108, 146-153 (2016).

40. Bae, S., Wong, H. L., Tie, J., Desai, J., Field, K., Kosmider, S. et al. Impact of diabetes status and medication on presentation, treatment, and outcome of stage II colon cancer patients. J. Cancer Epidemiol. 2015, 189132 (2015).

41. Ait Ouakrim, D., Dashti, S. G. \& Win, A. K. Response: aspirin, ibuprofen, and the risk for colorectal cancer in Lynch syndrome. J. Natl Cancer Inst. 108, djv385 (2016).

42. Okura, Y., Urban, L. H., Mahoney, D. W., Jacobsen, S. J. \& Rodeheffer, R. J. Agreement between self-report questionnaires and medical record data was substantial for diabetes, hypertension, myocardial infarction and stroke but not for heart failure. J. Clin. Epidemiol. 57, 1096-1103 (2004).

43. Dey, A. K., Alyass, A., Muir, R. T., Black, S. E., Swartz, R. H., Murray, B. J. et al. Validity of self-report of cardiovascular risk factors in a population at high risk for stroke. J. Stroke Cerebrovasc Dis. 24, 2860-2865 (2015).

44. World Health Organization. Global report on diabetes. (World Health Organization, Geneva, 2016). 